Research Article

\title{
Aerobics Movement Decomposition Action Teaching System Based on Intelligent Vision Sensor
}

\author{
Liwei Sun \\ Sports Department, Xi'an Aeronautical University, Xi'an, 710077 Shaanxi, China \\ Correspondence should be addressed to Liwei Sun; 201007008@xaau.edu.cn
}

Received 27 August 2021; Revised 14 October 2021; Accepted 26 October 2021; Published 13 November 2021

Academic Editor: Mu Zhou

Copyright ( 2021 Liwei Sun. This is an open access article distributed under the Creative Commons Attribution License, which permits unrestricted use, distribution, and reproduction in any medium, provided the original work is properly cited.

\begin{abstract}
With the development of the times, teaching has not only stayed between people, but also gradually developed into the teaching interaction between man and machine. In the past, the teaching form was relatively single and old. Based on the intelligent visual sensor, this paper develops an auxiliary teaching system for the decomposition of aerobics action and reasonably uses the Internet and algorithms to catalog a series of aerobics action systems into the system. The DTW dynamic motion matching algorithm of the system will recognize human actions more accurately. The system will feed back human actions to the system in real time based on human feature recognition. Then, after comparison, the system will display the standard posture of this action and the aerobics posture in the next step. Therefore, this system develops teaching not only in class, but everywhere. The system not only improves the teaching quality of aerobics, but also strengthens the physical quality of teenagers. It has a new understanding of the standardization of aerobics teaching. After the function of the system is complete, the system will be distributed to aerobics learners. In many feedback information, the average use satisfaction has reached about $80 \%$, which is a good performance index for the performance of the system itself.
\end{abstract}

\section{Introduction}

The physical health of students has gradually become the focus of public attention, and the concept of putting health first is thoroughly implemented. Effectively strengthen school physical education and encourage students to actively participate in sports activities. The National Standards for Student Physical Fitness and Health issued by the country clearly requires universities to conduct physical fitness tests on students every year. The "health quality and standards" of students at all levels improves people's living standards and raises health awareness, which is essential to promote the development of physical fitness testing systems. After meeting the needs of people's lives, people will pay more attention to physical fitness. With the rapid development of technologies such as networks, computers, and digital communications, other countries have entered the intelligent stage in the field of physical fitness testing. Start slowly to test the physical fitness test data, ignoring the complexity and the difficulty difference between the two in the data collection test process.
Computer-aided instruction (CAI) is a method and technology for discussing teaching content, the teaching process, and teaching of training students in a computerassisted conversation mode. CAI covers a wide range of computer technologies such as artificial intelligence, multimedia, and databases. It makes up for the shortcomings of traditional one-sided and one-sided teaching methods and provides students with a good personal learning environment. The use of CAI can effectively improve teaching quality, reduce learning time, improve the efficiency of teaching and learning in these two fields, and achieve the most appropriate educational goals. In the current teaching of aerobics, there is a problem that students cannot have an intuitive impression of the teaching content of movement, rhythm, music, difficulty, coordination, etc., and specific analysis of the structure of the movement process, as well as the overall perceptual understanding of the complete set of movements. CAI and intelligent vision sensors the combination can be effectively solved. The intelligent image sensor clearly displays the difficult points and key points of healthy gymnastics teaching from multiple 
angles. This makes aerobics education more vivid and effective, which helps to cultivate students' attention and enthusiasm for exercises. The design and development of CAI aerobics education project not only involves the transformation of aerobics teaching methods and teaching methods, but also affects the development of aerobics teaching style and teaching theory.

The research on the recognition of aerobics movements based on visual sensors is still in the theoretical stage of the laboratory, and we still need to make a certain amount of reference to other people's theories. Vision-Sensoren builds a high-speed intelligent vision sensor that integrates image acquisition and processing. The use of FPGA makes the image processing hardware, which greatly improves the speed of image acquisition and processing. The use of DSP enables the sensor to have its own CPU and perform some complex calculations. However, due to its greater flexibility, it is not suitable for general conditions [1]. Kettering realized the high-precision measurement of car body, continuously improved the level of visual inspection technology, studied the core part of the visual sensor, and analyzed the key technology of intelligent digital visual sensor, but the research did not enter a deeper theoretical discussion [2]. Zidek et al. use FPGA to implement the underlying image processing algorithm, which significantly speeds up the image processing speed and enhances the sensor performance. This design realizes the intelligentization and networking of the visual sensor and has great application value. However, because this design has developed too much quality and did not pay attention to the speed of transmission, the theory still needs to be studied [3]. Poornima et al. have carried out research on the related technology of intelligent digital vision sensor. The sensor uses fast template matching algorithm as the core and integrates the functions of image acquisition, feature coarse positioning, control decisionmaking, and digital information transmission to promote the detection system from analog. For signal transmission, the centralized processing mode of the central computer is converted to the digital signal transmission and the distributed processing mode of each node, which further improves the stability, real-time performance, and measurement accuracy of the system, but this theory is difficult to put into practice [4]. Shanthi studied the design of ODVS, embedded Linux ODVS system structure, ODVS video server, and ODVS application system software development, and elaborated on the specific application of the ODVS-based multitarget automatic tracking system, but the application cost is too high. There is no actual development value [5]. Suh et al. use linear scanning technology to obtain the scanning information of the object and cluster it into laser images according to the structural characteristics of the scanning information. The color vector of the laser image is extracted according to the storage arrangement method, and the edge structure of the object to be recognized is obtained through the nonlinear convolution operation to realize the edge detection of the laser image. Finally, the machine vision and laser image information point matching method is discussed. Based on the symmetry feature detection principle, the matched false information points are eliminated, and the remaining information points are output as the recognition result, but the final detection result does not reach the expected theoretical effect [6]. Harfmann proposed the use of MB-LBP texture features combined with radial basis functions as the kernel function SVM for face recognition and conducted a number of comparative experiments on the common Yale and ORL data sets. The experimental results show that the algorithm can be very good. Complete face recognition of intelligent robot vision. For face images with large illumination changes, reducing the number of iterations reduces the time complexity of face recognition, but it reduces the accuracy of the algorithm [7].

The design of this system is aimed at the shortcomings of actual aerobics teaching courseware and has developed functional modules including online courseware learning, online courseware arrangement, exchange forums, in-station message exchange, and content search. In the design of the system, this article describes and recognizes the movement characteristics of the human body and feeds the information back to the system, so that the system can provide real-time guidance and teaching for aerobics learners. The system's DTW dynamic action matching algorithm more accurately recognizes human actions. The system is based on human body feature recognition and feeds the human actions back to the system in real time. Then, the system will display the standard posture of this action after comparison and will perform the next step of bodybuilding. The posture will also appear, and the system has achieved a breakthrough in remote and man-machine teaching.

\section{Introduction to the Aerobics Movement Recognition Theory Method}

2.1. Theories of Body Movement Recognition Methods in Aerobics. Through training and learning to recognize aerobics movements, the computer can understand the meaning of a person's movements in a specific scene in the video. Figure 1 shows several basic processes of aerobics movement recognition, including information acquisition, preprocessing, representation, and description, and the two most important parts of classification and recognition are feature extraction and description and classification and perception. Both of these processes convert complex video data types into attributes in the form of arrays, vectors, and values containing motion data and then use classifiers to identify and classify these attributes [8-10].

The characterization and modeling of human motion is an important step in the process of perceiving human motion. The source video data consists of a series of continuous images, and each image contains a point of RGB value. Only by correctly mapping the source data can the granularity, and sensitive attributes contained in the data be produced. The current behavior methods can be divided into human body structure models, from the perspective of the structure and characteristics of motion, according to the human body model and behavior of motion.

Smart vision sensor is a highly integrated microminiature machine vision system. It is the fastest growing new technology in the field of machine vision in recent years. It 


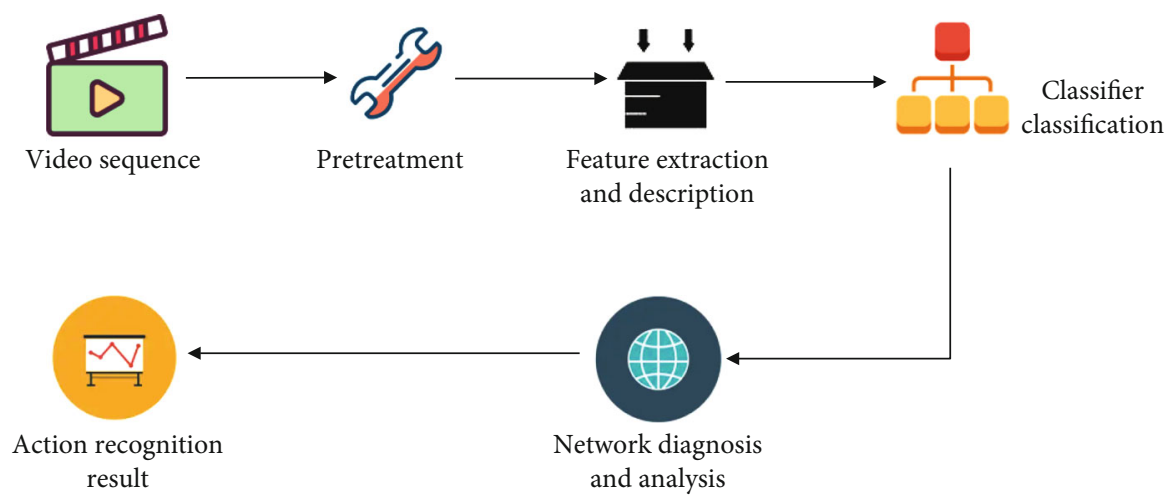

FIgURE 1: The basic process of human action recognition.

integrates image acquisition, processing, and communication functions into a single camera, thereby providing a machine vision solution that is multifunctional, modular, highly reliable, and easy to implement [11].

Pattern matching is one of the most common basic pattern recognition algorithms, refers to the alignment of two images. Image matching technology for the same target (or multiple images) in space is widely used in navigation, aerospace technology, and other industries and fields. Common gray-scale image matching methods such as target recognition and scene intrusion include gray-scale matching methods and feature-based matching methods. Gray matching method is generally point-to-point matching, so the relative accuracy is relatively high, and it is very sensitive to changes in interference $[12,13]$. When the external light changes, the detection result will be affected. At the same time, it is very complicated from a computational point of view, and rotation, deformation, and occlusion are all subtle. The selected attributes include points, lines, borders, and textures. Resist external light changes by isolating certain characteristics. Scale changes and rotation changes then use these features to match methods to make it stable. In the coordinate distribution in the image, first preset the exposure of a coordinate point $(x, y)$ in the image at time $t I(x, y, t)$ so that $u(x, y)$ and $v(x, y)$ can be regarded as points $(x, y)$ when the motion decomposition vectors in the $x$ and $y$ axis directions are decomposed, respectively. In a very short time differential $d t$, the point $(x, y)$ is shifted to the pixel coordinate $(x+d x, y+d y)$, and $d x=u d t, d y=v d t$, which represents the relationship between the spatial gray scale of the image and the optical flow velocity [14]. Table 1 is a list of parameters taken under different video sensors under the matching method.

In the optical flow constraint equation, both $u$ and $v$ are unknown. And there is only one constraint equation, so the luminous flux cannot be uniquely determined. Other constraints must be added to define $u$ and $v$. The introduction of different constraints will lead to different methods of calculating luminous flux [15]. The most classic is the gradient optical flow algorithm, also known as the differentiation method. Most of them are based on the gradient function of the gray image to get the motion vector of each pixel in the image. In the process of image template matching, due to some perspective distortions between the image to be matched and the template image, there are some partial and complete nonmatching phenomena. How to filter out these situations and how to measure the difference between the image to be detected and the template similarity of the distance matrix $D$ are as follows:

$$
\begin{aligned}
& Q=q_{1}, q_{2}^{\cdots \cdots \cdots}, q_{n} \\
& C=c_{1}, c_{2}, \cdots \cdots \cdot c_{n}, \\
& d\left(q_{1}, c_{1}\right) \quad d\left(q_{1}, c_{2}\right)^{\cdots \cdots \cdot} d\left(q_{1}, c_{n}\right), \\
& D=d\left(q_{2}, c_{1}\right) d\left(q_{2}, c_{2}\right)^{\cdots \cdots \cdot} d\left(q_{2}, c_{n}\right), \\
& d\left(q_{n}, c_{1}\right) d\left(q_{n}, c_{2}\right)^{\cdots \cdots} d\left(q_{n}, c_{n}\right) .
\end{aligned}
$$

The path of the curve is defined as follows: at two distances of different time series $D$, the path of curve $W$ is defined as a series of continuous matrix elements with different relationships between time series:

$$
W=\left\{w_{1}, w_{2}, \cdots \cdots w_{n}\right\} .
$$

The curved path satisfies the following constraints:

(1) Boundedness

$$
\operatorname{Max}(m, n) \leq k \leq m+n-1
$$

(2) Boundary conditions

$$
w_{1}=D(1,1), w_{k}=D(n, m)
$$

(3) Continuity

$$
w_{1}=D(i, j), w_{k-1}=D(O, P), I-O \leq 1, J-P \leq 1
$$

(4) Monotonicity

It is not allowed to appear at the same time.

$$
I-O \leq 1, J-P \leq 1 .
$$


TABLe 1: Comparison of detection at different width thresholds.

\begin{tabular}{lcccc}
\hline Width threshold & Fixed threshold $(5$ pixels $)$ & Template width of $1 / 3$ & Template width of $1 / 2$ & Template width $2 / 3$ \\
\hline Foreign body $(\leq 2 \mathrm{~cm})$ & 6589 & 5489 & 5784 & 5995 \\
Foreign body $(2 \sim 8 \mathrm{~cm})$ & 1520 & 1621 & 1425 & 1236 \\
Foreign body $(>8 \mathrm{~cm})$ & 1868 & 1896 & 1758 & 1851 \\
\hline
\end{tabular}

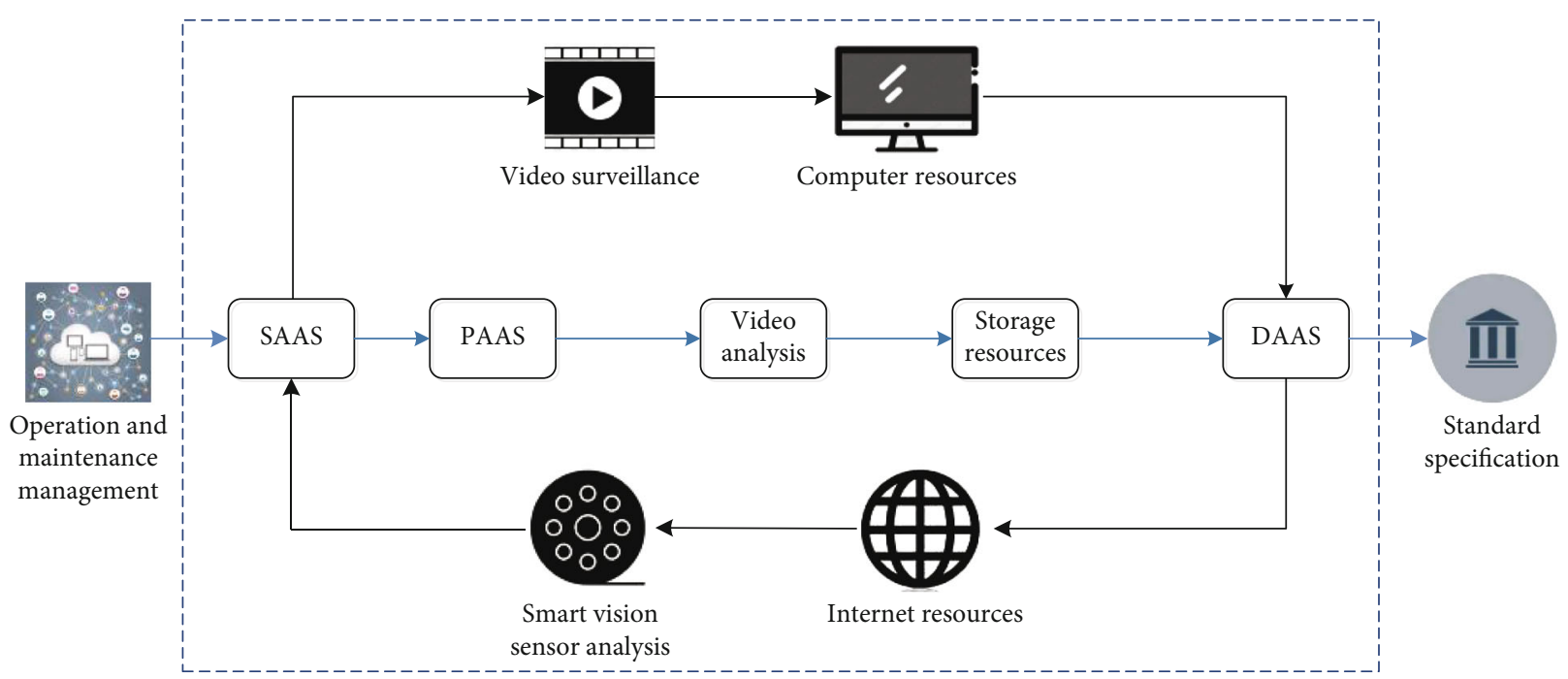

FIGURE 2: Framework diagram of the action collection system.

Therefore, after the above definition, find the iterative formula of the optimal path.

The motion recognition of aerobics is developed on the basis of intelligent visual sensors, using the combination of network real-time monitoring and visual sensors. The framework diagram designed is shown in Figure 2.

As an aerobics framework system, there are three main users in the aerobics teaching system: student users, teacher users, and management users. Among them, the main purpose of student users is to complete the study and review of the courseware; feedback the corresponding information to the teacher according to the mastery of the courseware; communicate with classmates and teachers. Learning experience or problems encountered in the study.

2.2. Body Movement Characteristics of Aerobics. Human body characteristics are the description of the appearance of human motion. Usually, the region of the moving human body should be identified in some key frames in the image sequence, individual textures, borders, contours, and other information [16, 17]. It is a feature model that calculates behavioral features and attributes to be measured. Pattern spacing is used to identify human behavior. This method can combine most of the image sequence data shown in Figure 3 for aerobics contour sequence. In order to describe the motion of the human body as a whole, Bobick and his colleagues superimposed the contours of the human body area to create a motion energy image (MEID) that can show the effect of motion and use dynamic functions to create motion to reflect the execution of the human body. The historical image of time (MHI) shows the generalization of recognition of human behavior. This method has a certain degree of durability as the shooting angle changes. Similarly, Huang Feiyue and others also perceive behaviors that have nothing to do with vision. It also uses cameras with different perspectives to capture the shape of the human body and calculate the "containment form."

There are six scientific methods to improve your body: weight-bearing exercise, interval training, repetitive exercise, continuous exercise conversion training, and comprehensive training. Choosing a comprehensive training method is a good way to develop a balanced body. This kind of aerobics exercise uses a comprehensive training method. Combine repetition and continuous exercises to organize student exercises. The overall content of the training is shown in Table 2.

The main content of the exercise program is self-made aerobics combined with strength exercises and flexibility exercises, and self-made aerobics uses low-impact actions and high-impact actions to be mixed and edited. The tenweek training is divided into three stages, the first stage, the second stage, and the third stage. In the three stages, the exercise load intensity will gradually increase, and the training will be carried out comprehensively, and the result is shown in Figure 3 [18].

The appearance of human body characteristics includes all the processes of human movement, which can provide complete information for action recognition, and the calculation process is relatively simple. However, the appearance of the human body depends on the image processing, such as precise background removal effect, too much positioning 


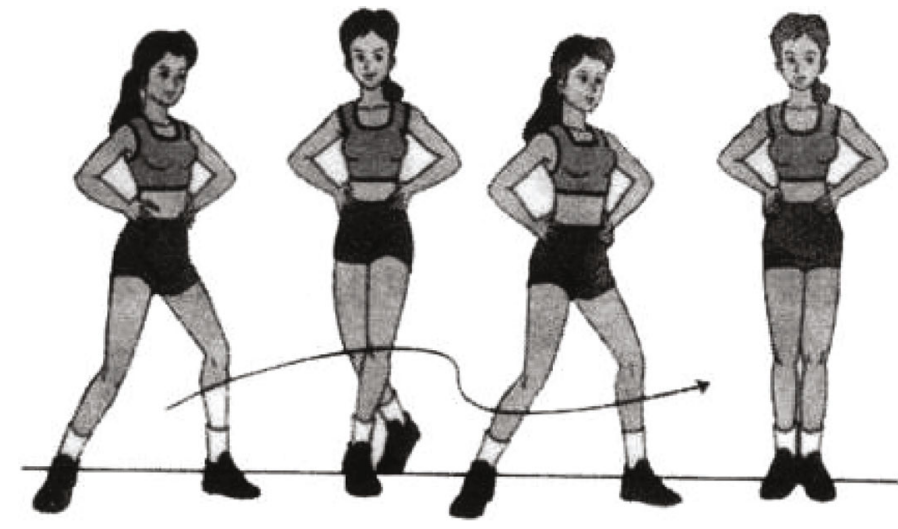

Figure 3: Schematic diagram of aerobics.

TABLE 2: The overall content and schedule of training.

\begin{tabular}{lccc}
\hline Stage & First stage & Second stage & Third stage \\
\hline \multirow{2}{*}{ Main activity content } & 1. Warm up & 1. Warm up & 1. Warm up \\
& 2. Slow-paced aerobics contact & 2. Midrhythm aerobics contact & 2. Fast-paced aerobics contact \\
Time & 3. Quality exercises & 3. Quality exercises & 3. Quality exercises \\
& 3 weeks, 3 lessons per week. & 3 weeks, 3 lessons per week. & 4 weeks, 3 lessons per week. \\
& 45 minutes per class & 45 minutes per class & 45 minutes per class \\
\hline
\end{tabular}

and tracking of moving targets. When the background environment is complicated, the viewing angle will change, which in turn affects the description of the action $[19,20]$. In order to implement a more systematic and reasonable teaching method and in order to verify the effectiveness of visual representation training in the early stage of aerobics skill learning, this article adds visual representation to the learning of aerobics skills.

2.3. DTW Dynamic Matching Action Recognition Algorithm. Whether the stereo matching algorithm can achieve better results mainly depends on three factors, including matching primitives, matching criteria, and matching algorithms. Among them, the stereo matching algorithm is a crucial part. The stereo matching algorithm is actually a process of finding the optimal solution, which determines the parallax of the pixel by solving the extreme value of an energy cost function. The stereo matching algorithm can realize different classifications according to different standards. Classification based on matching primitives can generally be divided into local-based stereo matching, global-based stereo matching, and feature-based stereo matching. Based on the size of the optimization range, stereo matching algorithms can be divided into local stereo matching and global stereo matching. Based on the density of the generated disparity map, it can be divided into sparse disparity matching and dense disparity matching. This article introduces several commonly used stereo matching algorithms, including the following: feature-based stereo matching, local stereo matching, and global stereo matching [21]. The factors affect the matching algorithm, from a large perspective, and the selected language has a great impact on the efficiency of the algorithm.
Generally speaking, more time and space were required to use the higher-level language. In addition, the quality of the code produced by different compilers is different, which will also have an impact on the efficiency of the algorithm.

The feature-based stereo matching algorithm refers to the disparity obtained by matching some features in the image. The features in an image usually involve points, lines, and geometric regions. The point feature is the most important feature. When using feature point matching, the feature point needs to be extracted before matching, and the extracted feature point must be clearly distinguishable from other pixels, so as to ensure the accuracy of parallax. This matching method is simple to implement, fast in matching speed, and has good real-time performance. However, since the matching effect is mostly dependent on the selection of feature points, the requirements for feature point selection are very high, and the effect is not ideal in some areas with slow gray-scale changes or weak texture areas. Since the number of feature points is small, the disparity map obtained is a sparse disparity map, so after this kind of matching, an interpolation algorithm is often needed to obtain a denser disparity map [22].

This system uses the DTW algorithm to locate and describe the human body features. The system's positioning is compared with the actual human body features, and then, the system will make corrections to give the correct selfcontained algorithm.

$$
\begin{aligned}
& T=\{T(1), \cdots, T(m), \cdots, T(M)\}, \\
& R=\{R(1), \cdots, R(n), \cdots, R(N)\} .
\end{aligned}
$$


It can be expressed as follows:

$$
\begin{aligned}
& T(M)=\left\{\theta_{t 1}, \theta_{t 2}, \cdots, \theta_{t x}\right\} \\
& R(N)=\left\{\theta_{r 1}, \theta_{r 2}, \cdots, \theta_{r x}\right\}
\end{aligned}
$$

According to formula (12), perform TFS code conversion on these 100 peak responses, where $r$ represents the address number, $r i$ is the peak response of the $i$-th address number, $t i$ is the input time of the $i$-th address number of the neural network, and $T$ is the expectation time of the last neuron input. In the feature sequence, the algorithm of the system is as follows:

$$
\begin{aligned}
P & =\left(k_{i}, l_{i}\right), i=1, j=1, \cdots, M, \\
k_{1} & =l_{1}=1, \\
k_{M} & =l_{M}=M, k_{i}<k_{i+1}, l_{i}<l_{i+1} .
\end{aligned}
$$

From this, it can be deduced that the distance difference between the human body feature and the system algorithm is shown in the following formula:

$$
\begin{aligned}
d\left[T\left(l_{j}\right), R\left(k_{i}\right)\right] & =\sum_{n=1}^{x} k_{n}\left\|\theta_{r n, l_{j}}, \theta_{t n, k_{j}}\right\|, \\
D[T, R] & =\sum_{k_{i}, j_{i} \epsilon P} d\left[T\left(l_{j}\right), R\left(K_{i}\right)\right], \\
\operatorname{DTW}[T, R] & =\min D[T, R], \\
\underset{E S}{\longrightarrow} & =\left(S_{X}-e_{x}, s_{y}-e_{y}\right), \\
\underset{E H}{\longrightarrow} & =\left(h_{X}-e_{x}, h_{y}-e_{y}\right) .
\end{aligned}
$$

The DTW algorithm is used to optimize the recognition response of the algorithm in recognizing human body aerobics actions. When the human body makes a series of exercise cycles, the simplified diagram of the optimal path of the system's feedback algorithm is shown in Figure 4.

Among them, the abscissa is the time series of the characteristic matrix. The name is the standard bone data timeline. The dotted line represents the optimal matching path, that is, the path with the greatest similarity. The weighted Euclidean distance is used to calculate the appropriate route in the area to calculate the similarity of the nodes in the graph. According to the formula, find the most similar time the sequence coordinates are compared to find the most suitable path as shown in the figure above [23].

\section{Aerobics Teaching Assistant System and Simulation}

3.1. Teaching System Framework. The technical architecture of the system can be divided into five layers. The Kinect data acquisition layer uses the Kinect smart camera to collect the depth images of the human body, human bone images, and human color images and transmits these data to the layout algorithm processing layer as video stream data. The algo-

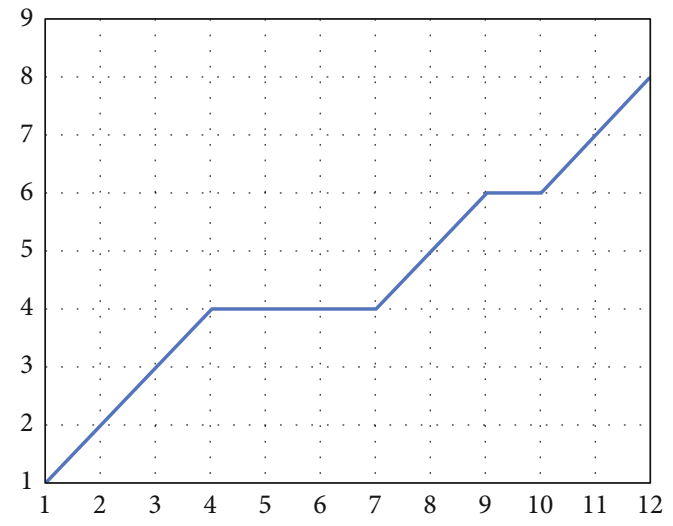

FIgURE 4: DTW shortest path diagram.

rithm processing layer filters this data. For DTW data and model matching, the processed bone node data is matched to the motion perception layer, and the results and results of the motion detection are displayed on the application layer. The frame structure of the system test system is shown in Figure 5.

Action recognition is a process of judging continuous events. In actual applications, in order to achieve the purpose of recognition and improve the efficiency of the task, it is necessary to constrain the execution point and execution interval of the judgment to avoid purposeless and repetitive recognition. At the same time, an important prerequisite for action recognition in practical applications is real-time detection of human action areas. In addition, in view of the importance of the action feature extraction step in the current action recognition process, visually presenting the action features extracted by different feature extraction algorithms for action recognition as a reference is also an important aspect in the application of action recognition [24, 25]. Based on the above observations, the action recognition application based on the service robot platform selects three starting points: exploring the effects of related action feature extraction algorithms, detecting human movements in real time, and recognizing human movements in a certain time interval. In order to accomplish the above goals, this chapter will design the software interface module, video control module, feature extraction module, and feature analysis module of the motion recognition application system to realize the experiment of motion recognition application; at the same time, use the motion recognition application software system to select applications experiment with scenes to analyze related algorithms.

As a CAI system, aerobics teaching system has three users: student users, teacher users, and management users. The main purposes of student users are as follows: to complete the learning and review of courseware, to give feedback corresponding information to teachers according to the learning and mastery of courseware, and to exchange learning experience or problems encountered in learning with classmates and teachers. The main purposes of teacher users are as follows: compiling new courseware content, improving the content of courseware through students' feedback information, answering questions to students, and 


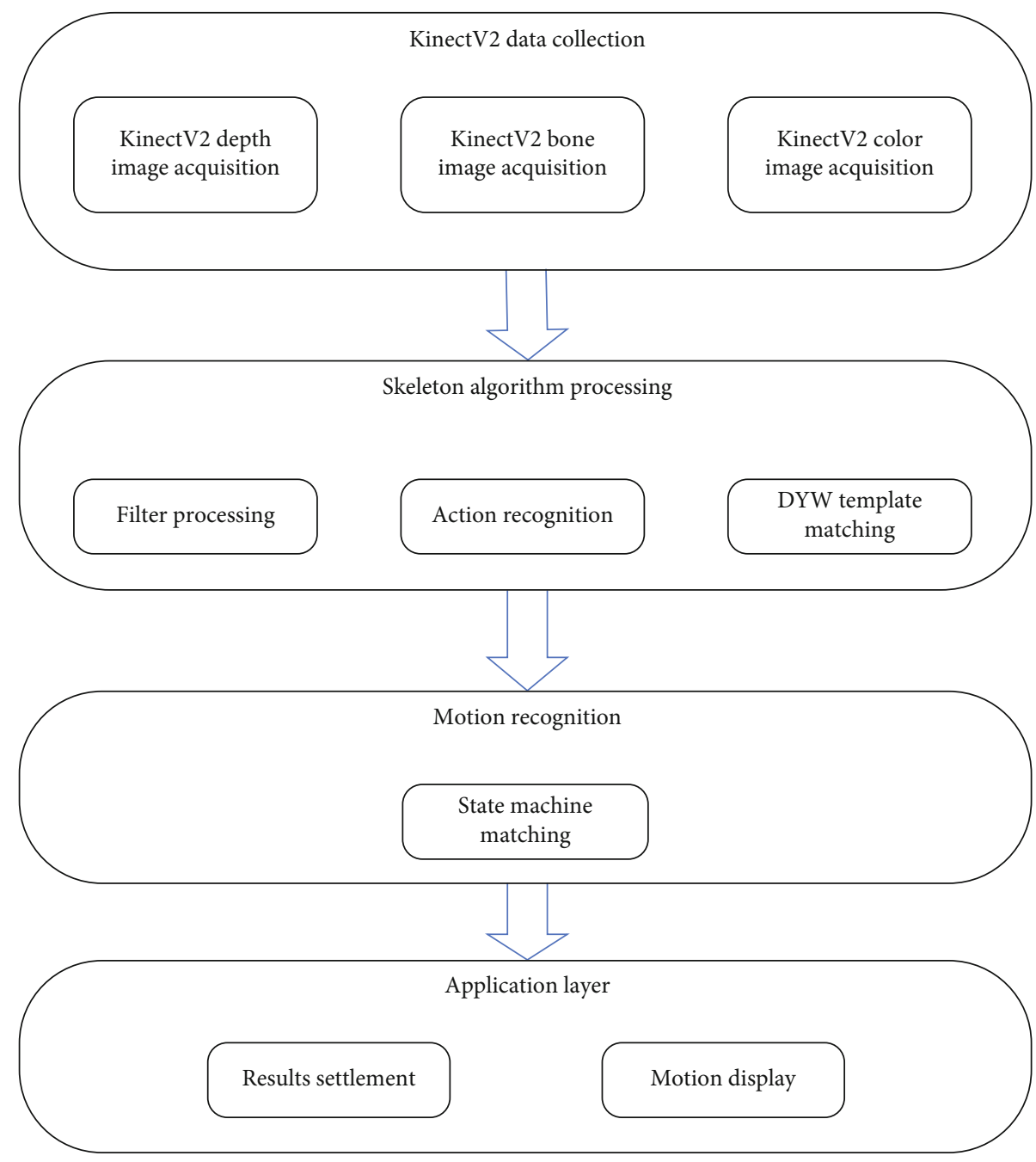

FIGURE 5: The frame structure of the physique test system.
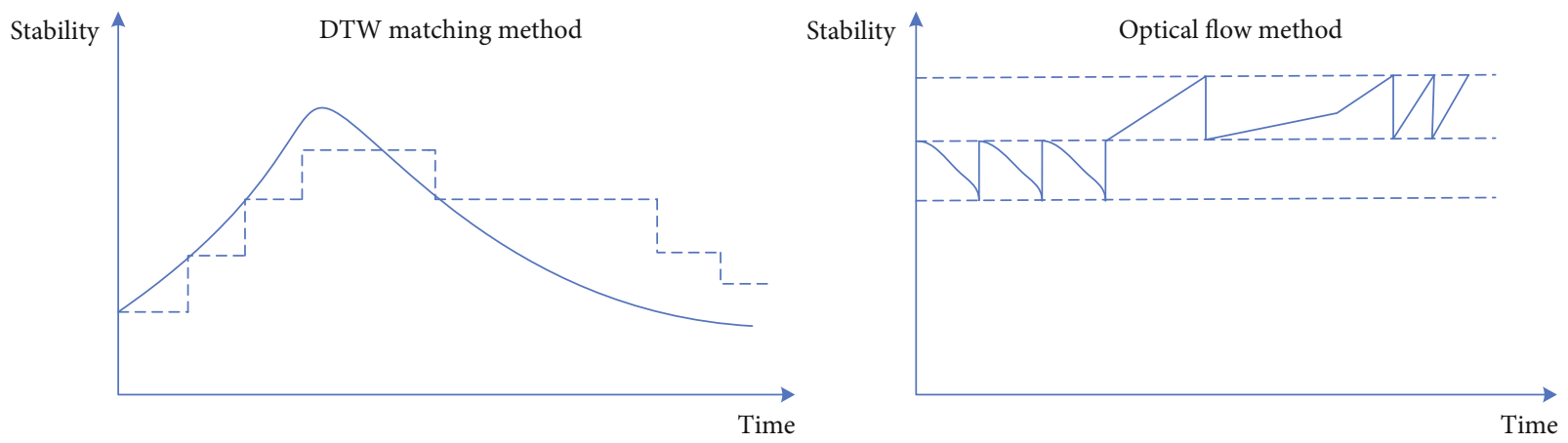

Figure 6: System stability comparison chart.

exchanging teaching experience with each other. The main purpose of managing users is to maintain system parameters and manage system users. The following systems use different methods for simulation experiments. The stability comparison of the simulation system is shown in Figure 6.

3.2. Calisthenics Action Recognition Experiment. In the algorithm based on feature point matching, the extraction of feature points is the foundation work. In order to realize the purpose of analyzing human motion, the extracted feature points must be pixels that can characterize human motion. According to the theory of topological vision, early human vision starts from a wide range of properties, and topological structure can be used as a description of this property. Among them, the human skeleton is of great significance for describing the three-dimensional information 
and topological structure of the human body, so this paper selects the human skeleton points as the matching feature points [26, 27]. First, extract the skeleton of the binarized image of the target detection. The main idea of skeleton extraction is to set the refinement conditions in advance and traverse the pixels in the input binarized image in turn to determine whether each point meets the setting. If the conditions are met, then mark. After the entire image is traversed, all the marked points are finally deleted. The first pixel stripping is completed, and then, the above process is repeated until the entire image no longer changes. What is obtained at this time is the target skeleton diagram. The algorithm maintains the topological structure of the target area, while reducing the complexity of subsequent processing. Tensor Flow is a second-generation artificial intelligence learning system developed by Google for machine learning and deep neural network research. Its name comes from its operating principle, that is, by constructing a data flow graph, data of tensor type is constructed. Transfer calculations in the flow graph.

When performing action recognition, this paper uses a support vector machine classifier to classify the gradient feature transformation characterization operator based on decoding. Among them, the decoding algorithm uses the interface function provided by the VLFeat library, and the support vector machine uses the interface function provided by the LibSVM library. Decoding algorithm is a process of using a specific method to restore the digital to the content it represents or to convert electrical pulse signals, optical signals, radio waves, etc. into the information, data, etc. it represents. Decoding is the process by which the receiver restores the received symbols or codes to information, which corresponds to the encoding process. Although the gradient feature can only accurately segment the relatively fixed action of the visual scene, in order to verify its temporal and spatial representation characteristics of the action, in addition to the KTH and Weizmann databases, the experiment also selected the UCF sports database with a certain change in the scene perspective as a benchmark [28].

For the three distinct movements of squatting, opening and closing, and standing, the recognition rate has reached more than $98 \%$; for the two similar actions of standing and opening and closing, the recognition rates are only $88.1 \%$ and $85.5 \%$. Squat is partly similar to one-knee squat and small jump, and the recognition rate is $90.2 \%$. The average recognition rate of algorithms based on gradient transformation features in the KTH database is $94.37 \%$. Table 3 compares the average recognition rates of different methods.

From the effect of action recognition, compared with other popular methods, the algorithm in this paper has strong competitiveness in recognition rate. One of the important reasons is that the algorithm efficiently extracts the spatial features of the gradient and deeply explores the temporal and spatial relationships of the spatial features of the gradient. At the same time, the settings of the default parameters have also been verified by many experiments [29].

This chapter conducts experiments on the matching algorithm network model constructed on the DTW data
TABLE 3: Comparison of average recognition results on KTH using different methods.

\begin{tabular}{lc}
\hline Method & Average recognition rate (\%) \\
\hline Derpanis etc. & 88.96 \\
Cao etc. & 91.25 \\
Laptev etc. & 90.16 \\
Le etc. & 90.47 \\
The algorithm of this paper & 93.58 \\
\hline
\end{tabular}

set. Figure 7 shows the matching algorithm model constructed in this paper under the DTW data set, using only the original gray data and adding the frame difference channel to the human body action recognition accuracy curve in the figure, the solid line represents the matching algorithm model constructed in this paper without the frame difference channel, and the dotted line represents the matching algorithm model with the frame difference channel added. After adding the frame difference channel, the network reached a high accuracy rate in the first few iterations of training, the error dropped quickly, and it soon began to converge. Under the action of the frame difference channel, the network quickly captured the human behavior characteristics, while the network that did not use the frame difference channel had to find the available features in the original image by itself and gradually learned the human behavior characteristics after dozens of rounds of training [30]. Introducing a visual attention mechanism into the DTW algorithm can improve the accuracy of human behavior recognition.

3.3. Aerobics Experiment Simulation. The test subjects were tested before the experiment, and the subjects were divided into intervention groups and control groups according to natural classes when there were no significant differences in the indicators of the subjects. The intervention group performed the training specified in the system exercise program of this article within ten weeks, and the control group did not do any intervention training except for physical education classes in accordance with the school syllabus. After the experiment was completed, all the experimental subjects were tested again, and the indicators of different groups were compared and analyzed, and a conclusion was reached [31].

The main content of the exercise program is self-made aerobics combined with strength exercises and flexibility exercises, and self-made aerobics uses low-impact movements and high-impact movements for mixed creation. The ten-week training is divided into three stages, the first stage, the second stage, and the third stage. The exercise load intensity of the three stages will gradually increase. Comprehensive training: the main content of this program is selfcompiled aerobics with a total of $10 \times 8$ movements. The rhythm is controlled by system music or passwords. The movements are novel, but simple and easy to learn. Specific action content: all students stand in four horizontal rows. The first 8 beats are in situ running motions, with the arms swinging naturally; the second 8 beats are foot-to-ground motions with the arms extended to the side; the third 8 beats are a "one" step, and the arms are forward and natural, 

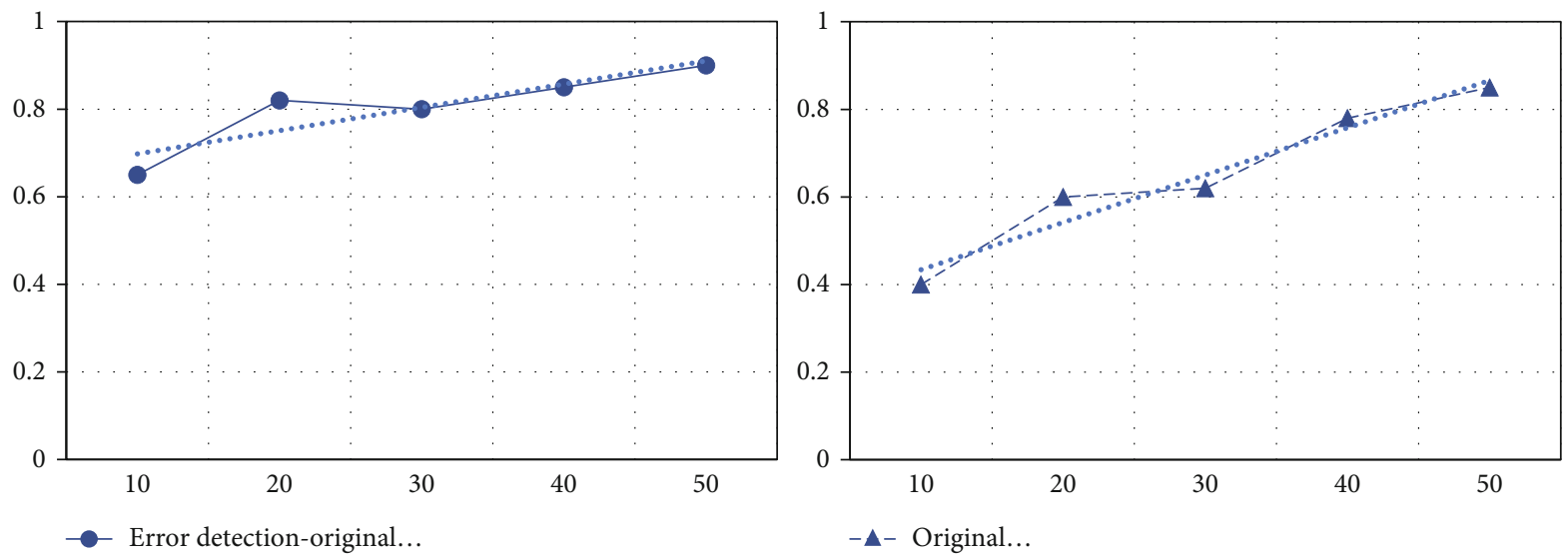

FIgURE 7: Recognition accuracy curve of dual-channel 3D convolutional neural network model.

respectively. Put down the action; the fourth 8 th beat is a side step, and the arms are opened and closed according to the beat; the fifth 8th is a "V" step, and the arms follow the feet to make a pose; the sixth eight beats up step knee and leg movements; the seventh eight beats for kicks; the eighth eight beats for knees and legs jumps; the ninth eight beats for pony jumps; the tenth eight beats for running and jumping. This set of self-programming can jump continuously, and the system arranges the jump mode. The system uses passwords and music speed to control the exercise intensity [32].

According to the requirements of the syllabus and aerobics assessment and evaluation standards, the golden aerobics scoring standard is adopted. Due to the needs of this experimental study, the technical assessment is carried out by the way that the students do actions by calling out the password. The scoring content mainly includes six aspects: basic posture, movement proficiency, movement accuracy, movement range and strength, movement coordination, and movement rhythm and expressiveness. According to the grading rules, three professional teachers with aerobics first-level referee certificate will score the three classes under the same conditions and take the middle value of the three teachers as the final score. Use Polar meter to track and monitor students' exercise intensity during exercise, and record students' subjective movement feelings. The fourth stage: after the experiment is completed, the staff who organizes the pretest test will perform the postexperiment test of the various indicators on the experimental object.

The key to the exercise of sit-ups is the state action of situps. The ratio of the scores between the supine state and the sit-up state is b1:b2. Take a sit-up test cycle as an example. The full score of each sit-up exercise cycle is $m$. In actual conditions, $m$ is 100 points. Assuming that the tester has completed $n$ squats, the standing state similarity is $g$, and the sit-up state similarity is $k$, and the final score $S$ is obtained according to the formula.

These three experimental classes are composed of three groups: experimental group, interference group, and control group. The experimental group is arranged for the usual aerobics training through the aerobics movement decomposition teaching system designed in this article, while the other two groups are composed of coaches and self-

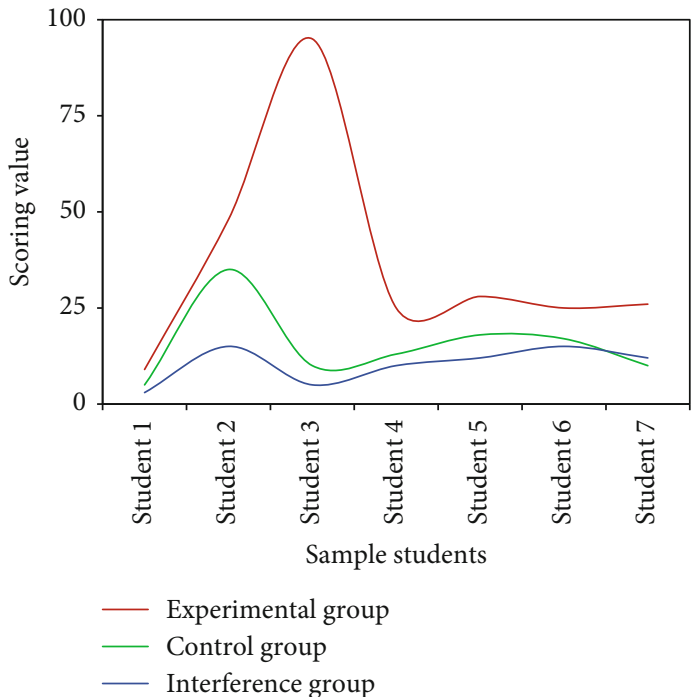

FIgURE 8: The scores of students' aerobics in different experimental groups.

learning. There are two ways to learn aerobics to learn aerobics movements. Figure 8 shows the scores of the above three groups.

Because aerobics can significantly increase the strength of the respiratory muscles, improve the elasticity of the lungs, increase and deepen the depth of breathing, and improve the efficiency and function of lung breathing. In the process of aerobic exercise, a lot of breathing air is required to breathe in. During exercise, the muscle contraction if surgery requires a lot of oxygen. The demand for oxygen increases, the number of breaths is more than normal, and the lungs contract more. Long-term exercise enhances the functioning capacity of the lungs.

The scheme is designed to monitor during training, and the combination of the two methods is used. It can be seen from Table 4 above that the exercise intensity gradually increases from the first stage to the third stage in the whole training process of the scheme. The data heart rate value in Table 4 shows that the exercise load of the scheme is medium, and the exercise sensation level of the athlete is 
TABLE 4: Comparative analysis of the final assessment results of students in the three experimental classes learning new technical movements.

\begin{tabular}{lcccc}
\hline & Average & Standard deviation & $t$ value & $p$ value \\
\hline Experiment 1 & 7.598 & 0.365 & 4.021 & $0<(0.05)$ \\
Experiment 2 & 8.598 & 0.289 & 4.215 & $0<(0.05)$ \\
Experiment 3 & 7.145 & 0.314 & 4.587 & $0<(0.05)$ \\
\hline
\end{tabular}

between 12 and15, indicating that the load intensity is reasonable, so the exercise scheme is reasonable. This size also shows that the exercise intensity is medium, and the relative intensity is about $40 \%-60 \%$. Relevant studies have shown that aerobics is a low-intensity and long-term exercise, the exercise intensity is medium or above, the heart rate range is $60 \%-85 \%$ of my maximum heart rate, the maximum heart rate $=200-$ self age, and the ages of junior two students are between 13 and 15 years old. Therefore, this sports program is fully in line with the characteristics of aerobics. This kind of training can enhance people's cardiopulmonary endurance, improve people's cardiovascular function, enhance people's vital capacity and heart function, and lose weight and fat.

\section{Experimental Results and Analysis}

4.1. Stability Analysis of Aerobics Teaching Action Decomposition System. System testing is the last stage studied in this subject, and it is also the most critical link to ensure the quality of system design. It requires the use of various testing methods to detect the completeness of system functions and the efficiency of system execution. Here, the system is inspected mainly through function test, performance test, and example test. The main purpose of functional testing is to judge whether the various modules and functions designed in the system analysis process can work normally and to find and solve the problems encountered in the process of coding and design. For the functional test of the system, the main focus is on the following aspects:

(1) User Login Function. Verify whether the user's login function is normal, whether the user can be redirected to the specified user interface, and whether illegal user login can be rejected.

(2) Courseware Learning and Feedback Function. Verify whether student users can browse courseware types normally and whether various contents provided by regular learning courseware can be fed back to teacher users normally.

(3) Courseware Layout Function and Feedback Query. Verify whether teacher users can normally edit courseware containing text, images, sound, and video content and whether the layout function of the courseware is perfect: verify whether the feedback information of student users on the courseware can be checked normally.
(4) User Management Function Verification. Verify whether the administrator user can normally add, delete, and modify various user categories and user information.

(5) Verification of the Communication Function. Verify whether the messages in the station can be sent and received normally, and verify whether the exchange forum can normally post and reply normally.

In accordance with the above test requirements, the login, use, communication, and management functions of the system were carefully tested one by one, and the problems that appeared in the test were corrected and improved to ensure the reliability of the system's operation. Figure 9 below is a comparison diagram of the system response time for the predicted stability and actual stability of the system.

Obviously, it can be seen from Figure 9 that in the comparison, the reaction time of the system in this article is not much different from the expected response time, which shows that the reaction time of the system still meets the expected requirements and expectations when the actual system is used. Generally speaking, due to the complexity of human movements, the accuracy of training-based motion detection methods is greatly affected by training samples, and the algorithm efficiency is low, but the detection accuracy is not affected by background changes. Motion detection based on gradient transformation projection has better reliability in practical application scenarios where the background changes little, but it has a poorer detection effect for motion video with large background changes.

4.2. Analysis of the Aerobics Teaching System in Actual Use. The aerobics teaching system of this article will be distributed to 100 aerobics learners, and 100 aerobics learners will learn aerobics by themselves or by multimedia. After one stage of learning feedback, Figure 10 shows the feedback of the above 100 users.

From a physiological point of view, these data change, students participate in a certain period of planned exercise training, and after a long period of exercise, the coordination ability of the receptors will gradually increase; therefore, the balance ability will increase; training makes the cerebral cortex excited during exercise state, and the neural connection speed of the cerebral cortex will become rapid and accurate, so the agility will be improved. However, in the aerobics exercise program, there is no systematic training content designed for balance quality and reaction speed, so there is no significant difference in the changes of related indicators. This system is only aimed at the systematic teaching of the decomposition teaching of aerobics movements. According to the satisfactory responses of the teaching experimenters, most users still have a relatively good evaluation of the system, with a satisfaction rate of about $80 \%$. It makes users more interested in aerobics training and more confident in their own learning ability. The purpose of adolescent fitness exercise is to promote the growth and development of the body and enhance the quality and health of the body. According to the law of growth and development of 

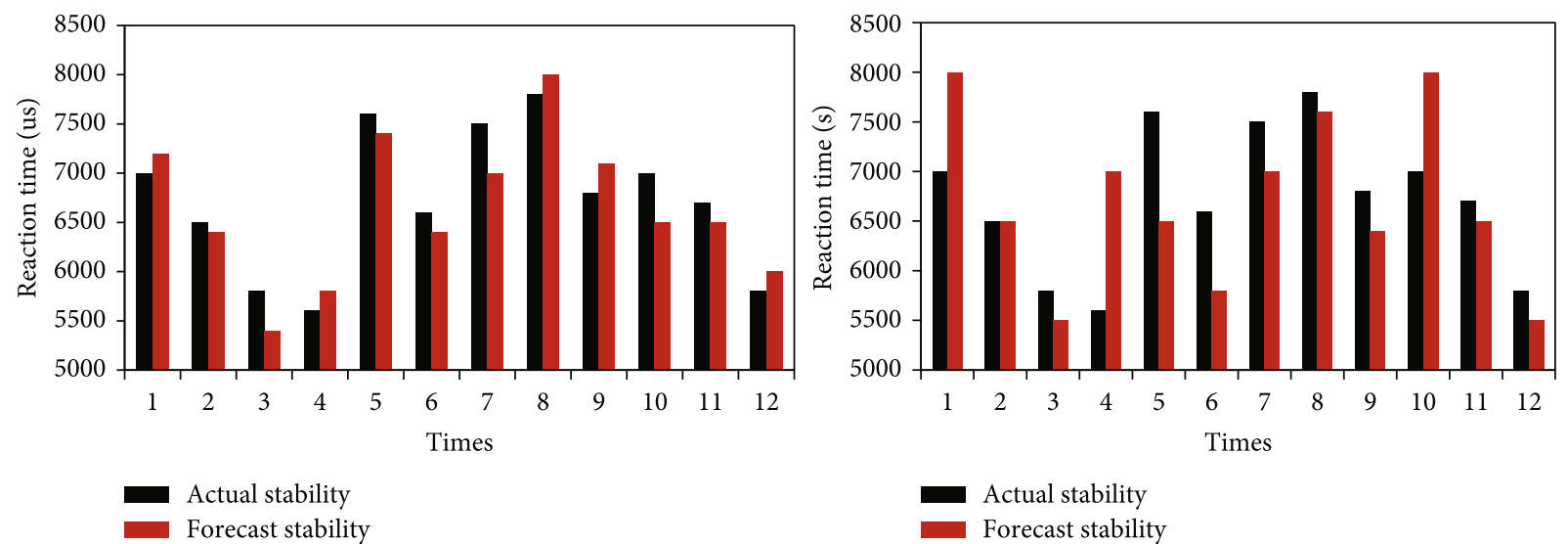

FIGURE 9: System response time comparison chart.
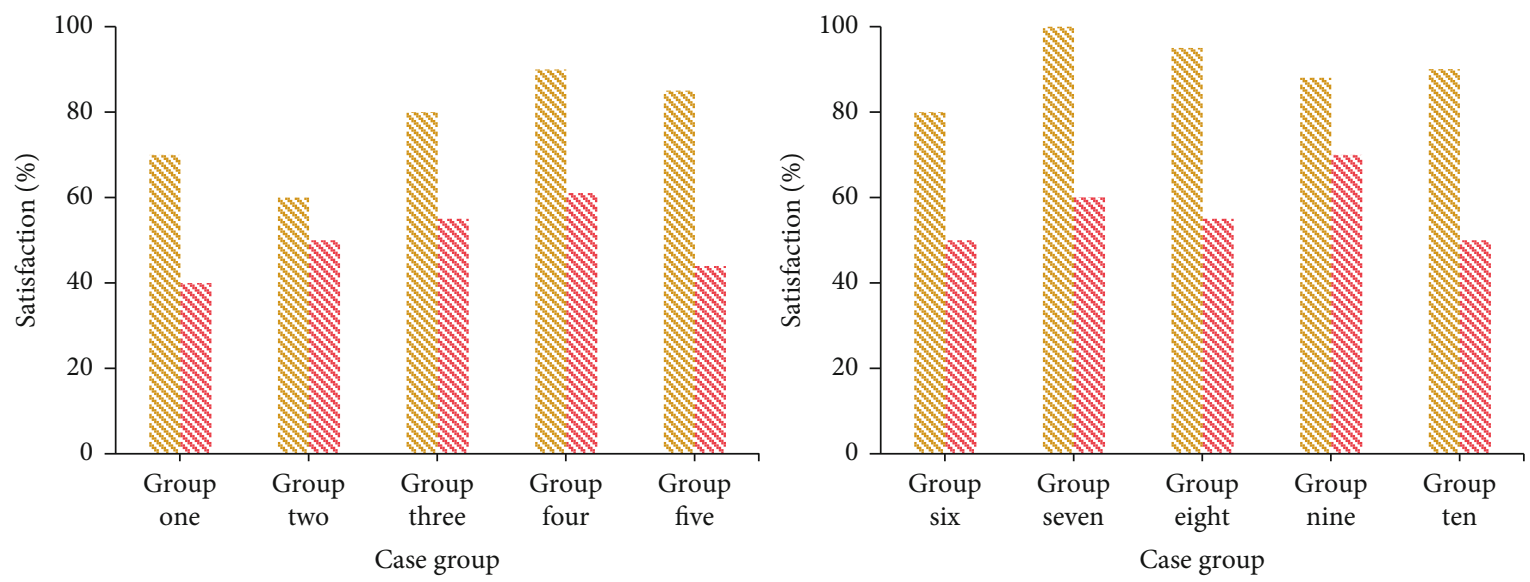

FIgURe 10: Use satisfaction survey.

adolescents, the main indicators that can effectively reflect the fitness effect are body shape, physical function, physical fitness, and other indicators. Therefore, the aerobics teaching system in this article effectively solves the social embarrassment of young people facing people before teaching. It is a good idea to reach an agreement between the interaction between man and machine and teaching.

\section{Conclusion}

Based on intelligent vision, this paper develops an auxiliary teaching system for the decomposition of aerobics movements. The aerobics teaching system based on the B/S structure has basically been designed and can be put into use. The design of this system is aimed at the shortcomings of actual aerobics teaching courseware and has developed functional modules including online courseware learning, online courseware arrangement, exchange forums, instation message exchange, and content search. The algorithm of the aerobics system is designed through the DTW matching algorithm, so that the fluency of the system meets the basic requirements in normal operation. In the design of the system, this article describes and recognizes the movement characteristics of the human body and feeds the infor- mation back to the system, so that the system can provide real-time guidance and teaching for the aerobics learners. After a later simulation of the system and a survey on crowd use, the average satisfaction of the system reached $80 \%$, and the level of aerobics learners has been greatly improved, indicating that the feedback on the use of the system is still relatively to a certain extent $\mathrm{OK}$. Although this system has realized the functions necessary for basic aerobics teaching, it still has a certain distance compared with a perfect CAI system. Moreover, the development of domestic aerobics teaching CAI has just started, and there will be some in the future. More and more excellent development ideas and development methods appear. To get a fully functional aerobics CAI system, more manpower and material resources need to be invested.

\section{Data Availability}

No data were used to support this study.

\section{Conflicts of Interest}

There are no potential competing interests in our paper. 


\section{Authors' Contributions}

The author has seen the manuscript and approved to submit to your journal.

\section{Acknowledgments}

This work was supported by the Project of Social Science Foundation of Shaanxi Province in 2020: "Research on the development path of aerobics dance in Shaanxi Province from the perspective of Healthy China" (No. 2020Q003).

\section{References}

[1] Bernd and Eckenfels, "Vision-Sensoren vereinfachen die einrichtung von pick-and-place-anwendungen: direkte kommunikation mit dem roboter," Elektro-Automation: Elektrotechnik + Elektronik Inder Industrie, vol. 71, no. 6, pp. 70-71, 2018.

[2] S. Lee, G. Tewolde, J. Lim, and J. Kwon, "Vision based localization for multiple mobile robots using low-cost vision sensor," International Journal of Handheld Computing Research, vol. 7, no. 1, pp. 12-25, 2016.

[3] K. Zidek, V. Vasek, J. Pitel, and A. Hosovsky, "Auxiliary device for accurate measurement by the smart vision system," $M M$ Science Journal, vol. 2018, no. 1, pp. 2136-2139, 2018.

[4] J. Poornima, J. Vishnupriyan, G. K. Vijayadhasan, and M. Ettappan, "Voice assisted smart vision stick for visually impaired," International Journal of Control and Automation, vol. 13, no. 2, pp. 512-519, 2020.

[5] K. G. Shanthi, "Smart vision using machine learning for blind," International Journal of Advanced Science and Technology, vol. 29, no. 5, pp. 12458-12463, 2020.

[6] J. Suh, S. You, S. Choi, and S. Oh, "Vision-based coordinated localization for mobile sensor networks," IEEE transactions on automation science and engineering, vol. 13, no. 2, pp. 611-620, 2016.

[7] B. Harfmann, "Ensuring safety through sensor solutions," Beverage Industry, vol. 107, no. 7, pp. 42-43, 2016.

[8] N. Rossol, I. Cheng, and A. Basu, "A multisensor technique for gesture recognition through intelligent skeletal pose analysis," IEEE Transactions on Human-Machine Systems, vol. 46, no. 3, pp. 350-359, 2016.

[9] M. Zhou, Y. X. Long, W. P. Zhang et al., "Adaptive genetic algorithm-aided neural network with channel state information tensor decomposition for indoor localization," IEEE Transactions on Evolutionary Computation, vol. 25, no. 5, pp. 913-927, 2021.

[10] G. I. Lee, C. M. Kang, S. H. Lee, and C. C. Chung, "Sensor fusion based on the particle filter and multi-rate Kalman filter," Journal of Institute of Control, vol. 23, no. 11, pp. 969980, 2017.

[11] M. Zhou, Y. M. Wang, Z. S. Tian, Y. Lian, Y. Wang, and B. Wang, "Calibrated data simplification for energy-efficient location sensing in Internet of Things," IEEE Internet of Things Journal, vol. 6, no. 4, pp. 6125-6133, 2019.

[12] M. Weigel, H. Fiedler, and T. Schildknecht, "Scoring sensor observations to facilitate the exchange of space surveillance data," Advances in Space Research, vol. 60, no. 3, pp. 531$542,2017$.
[13] J. W. Kim, Y. D. Jung, D. S. Lee, and D. H. Shim, "Landing control on a mobile platform for multi-copters using an omnidirectional image sensor," Journal of Intelligent \& Robotic Systems, vol. 84, no. 1-4, pp. 529-541, 2016.

[14] A. Kuzdeuov, M. Rubagotti, and H. A. Varol, "Neural network augmented sensor fusion for pose estimation of tensegrity manipulators," IEEE Sensors Journal, vol. 20, no. 7, pp. 36553666, 2020.

[15] E. Joshi, D. S. Sasode, N. Singh, and N. Chouhan, "Wireless sensor network application for precision agriculture," Research Today, vol. 2, no. 5, pp. 125-128, 2020.

[16] R. Verma and K. Srivastava, "Middleware, operating system and wireless sensor networks for Internet of Things," International Journal of Computer Applications, vol. 167, no. 11, pp. 11-17, 2017.

[17] X. Sun, A. Basu, and I. Cheng, "Multi-sensor motion fusion using deep neural network learning," International Journal of Multimedia Data Engineering and Management, vol. 8, no. 4, pp. 1-18, 2017.

[18] S. Hensel, "Machine vision als integraler bestandteil der automation," Elektrotechnische Zeitschrift, vol. 138, no. s5, pp. 4851, 2017.

[19] J. A. Lenero-Bardallo, P. Hafliger, R. Carmona-Galan, and A. Rodriguez-Vazquez, "A bio-inspired vision sensor with dual operation and readout modes," IEEE Sensors Journal, vol. 16, no. 2, pp. 317-330, 2016.

[20] L. Wang, W. Song, Y. Lan et al., "A smart droplet detection approach with vision sensing technique for agricultural aviation application," IEEE Sensors Journal, vol. 99, 2021.

[21] L. Chermak, N. Aouf, M. Richardson, and G. Visentin, "Realtime smart and standalone vision/IMU navigation sensor," Journal of Real-Time Image Processing, vol. 16, no. 4, pp. 117, 2016.

[22] F. Ortega-Zamorano, M. A. Molina-Cabello, E. López-Rubio, and E. J. Palomo, "Smart motion detection sensor based on video processing using self-organizing maps," Expert Systems with Application, vol. 64, no. Dec., pp. 476-489, 2016.

[23] Y. Wu, Y. Wang, W. Hu, and G. Cao, "SmartPhoto: a resourceaware crowdsourcing approach for image sensing with smartphones," IEEE Transactions on Mobile Computing, vol. 15, no. 5, pp. 1249-1263, 2016.

[24] B. G. Lee and M. L. Su, "Smart wearable hand device for sign language interpretation system with sensors fusion," IEEE Sensors Journal, vol. 18, no. 3, pp. 1224-1232, 2018.

[25] M. Behrens, K. Müller, J. I. Kilb et al., "Modified step aerobics training and neuromuscular function in osteoporotic patients: a randomized controlled pilot study," Archives of Orthopaedic and Trauma Surgery, vol. 137, no. 2, pp. 195-207, 2017.

[26] W. Zheng, "Experimental study on aerobics teaching model based on cognitive flexibility theory," Aerobics \& Fitness, vol. 1, no. 1, pp. 1-5, 2016.

[27] Y. Ma, "Cultivation of the ability of creating and arranging aerobics in physical education majors," World Scientific Research Journal, vol. 5, no. 9, pp. 88-93, 2019.

[28] S. Tonstad, P. Herring, J. Lee, and J. D. Johnson, “Two physical activity measures: Paffenbarger physical activity questionnaire versus aerobics center longitudinal study as predictors of adult-onset type 2 diabetes in a follow-up study," American journal of health promotion, vol. 32, no. 4, pp. 1070-1077, 2018.

[29] M. Ebrahimi, T. N. Guilan-Nejad, and A. F. Pordanjani, "Effect of yoga and aerobics exercise on sleep quality in women with 
type 2 diabetes: a randomized controlled trial," Sleep Science, vol. 10, no. 2, pp. 68-72, 2017.

[30] G. Ali Sholi, M. Ghanbarzadeh, A. Habibi, and R. Ranjbar, "The effects of combined exercises intensity (aerobics-resistance) on plasma cortisol and testosterone levels in active males," International Journal of Basic Science in Medicine, vol. 1, no. 1, pp. 18-24, 2016.

[31] W. Bei, "Research on body language in aerobics choreography and physical education based on network questionnaire," International Journal of Future Generation Communication and Networking, vol. 9, no. 4, pp. 207-218, 2016.

[32] N. Garrido, J. D. Silva, J. S. Novaes, M. S. Cirilo-Sousa, and G. R. Neto, "Effect of water aerobics on the quality of life, satisfaction, and perception of body image among elderly women," Journal of Exercise Physiology Online, vol. 19, no. 5, pp. 30-37, 2016. 